\title{
Quantum Logic Gates in Optical Lattices
}

\author{
Gavin K. Brennen, ${ }^{(1)}$ Carlton M. Caves, ${ }^{(1)}$ Poul S. Jessen, ${ }^{(2)}$ and Ivan H. Deutsch ${ }^{(1)}$ \\ ${ }^{(1)}$ Center for Advanced Studies, Department of Physics and Astronomy, \\ University of New Mexico, Albuquerque, NM 87131 \\ ${ }^{(2)}$ Optical Sciences Center, University of Arizona, Tucson, AZ 85721
}

(February 1, 2008)

\begin{abstract}
We propose a new system for implementing quantum logic gates: neutral atoms trapped in a very far-off-resonance optical lattice. Pairs of atoms are made to occupy the same well by varying the polarization of the trapping lasers, and then a near-resonant electric dipole is induced by an auxiliary laser. A controlled-NOT can be implemented by conditioning the target atomic resonance on a resolvable level shift induced by the control atom. Atoms interact only during logical operations, thereby suppressing decoherence.
\end{abstract}

1998 PACS numbers: 03.67.Lx, 32.80.Qk, 32.80.Lg, 32.80.Pj

Any computation is constrained by the physical laws governing the machine that carries out the operations. Conventional computers operate according to the laws of classical physics, but an entirely new class of computers is possible using physical components that are governed by the laws of quantum mechanics [1]. At the heart of quantum computation is the entanglement of many twostate systems (qubits), which form the register of the quantum computer. The requirements for creating and maintaining such a highly entangled state seem to be almost contradictory: the qubits must be strongly coupled to one another and to an external field to produce the conditional-logic operations for quantum computation, yet coupling to other external influences must be minimized because it leads to decoherence. Quantum error correction [2] and fault-tolerant computation [3] promise to defeat the deleterious effects of decoherence, but only if the coupling to the environment is sufficiently weak.

Several physical realizations of quantum computation have been proposed. One of the most promising is based on storing each qubit in the state of an ultracold trapped ion 迎. Ions interact strongly via their mutual Coulomb repulsion, thus allowing unitary manipulation of the qubits' joint state to be achieved with lasers [5]. Because of their charge, however, the ions interact strongly with the environment, giving rise to decoherence channels from technical noise sources [6]; possiblities for surmounting these problems are currently being explored [7. Elements of quantum computation have also been implemented in standard NMR apparatuses [8] and in cavity QED [9], but these schemes are at present difficult to scale to many qubits. Solid-state systems, including quantum dots [10 have also been proposed for realizing quantum computation, but the strong interactions that exist in a condensed-matter environment make decoherence a difficult problem. A recent proposal [11] to marry NMR techniques with silicon technology looks promising.

We propose here a new system for implementing quantum logic gates: trapped neutral atoms made to interact via laser-induced coherent electric dipole-dipole interactions. Such a system has two advantages: decoherence is suppressed because neutrals couple weakly to the environment, and operations can be performed in parallel on a large ensemble of trapped atoms, thus offering avenues for scaling to many qubits. The main source of decoherence is spontaneous emission, but this can be negligible if all manipulations are performed rapidly compared to the photon scattering rate. To see that this is possible, consider the following scaling argument. The photon scattering rate is $\Gamma^{\prime}=s \Gamma / 2$, where $s$ is the saturation parameter, proportional to the excited state population, and $\Gamma \sim k^{3}\left|d_{e g}\right|^{2} / \hbar$ is the spontaneous emission rate, $k$ being the wave number of the photon and $d_{e g}$ the dipole matrix element between the ground and excited states. For atoms spaced at distances small compared to the optical wavelength, retardation effects are negligible, and the level shift arising from the near-field dipole-dipole interaction scales as $V_{d d} \sim\left\langle d_{1}\right\rangle\left\langle d_{2}\right\rangle / r_{12}^{3}$, where $\langle d\rangle$ is the dipole expectation value and $r_{12}$ is the characteristic separation between the dipoles. For weak (nonsaturated) excitation $\langle d\rangle \sim \sqrt{s} d_{e g}$, so the ratio of interaction energy to scattering rate scales as $\kappa \sim V_{d d} / \hbar \Gamma^{\prime} \sim\left(k r_{12}\right)^{-3}$. Thus, if the atoms can be tightly confined to relative distances small compared to the wavelength, one can induce a coherent dipole-dipole interaction with negligible photon scattering. The central point is that the coherent level shift can be enhanced substantially through tight confinement, while the cooperative spontaneous emission rate cannot increase by more than a factor of two (the Dicke superradiant state) over that of an isolated atom. In addition, since the resonant dipoles can be turned "on" and "off" at will, atoms can be made to interact only during the conditional logic operations and not during single-qubit manipulations or during periods of free evolution, thereby reducing coupling to the environment.

As a concrete implementation, we consider here the use of neutral alkali atoms trapped in a far-off-resonance optical lattice, periodic potentials created by a set of interfering laser beams in which atoms are trapped via the ac-Stark shift [12]. By detuning the lasers very far from resonance, photon scattering is greatly reduced. Through 
a combination of near-resonance Sisyphus laser cooling and resolved-sideband Raman cooling [13], atoms can be prepared in the ground state of the potential wells. In a recent experiment, $\sim 10^{6} \mathrm{Cs}$ atoms were cooled in a two-dimensional optical lattice, with mean vibrational excitation $\bar{n} \approx 0.01[13$. Such atoms can be tightly confined, with an rms spread on the order of $\Delta x \approx \lambda / 50$ for reasonably deep wells, and are thus good candidates for inducing coherent dipole-dipole interactions. According to the discussion above, the ratio of the level shift to the linewidth is $\kappa=C / \eta^{3} \approx 500 C$, where $\eta=k \Delta x$ is the Lamb-Dicke parameter and $C$ is a number depending on the details of the geometry, to be determined below.

Consider a 3D optical lattice, detuned far to the blue of atomic resonance, with atoms trapped at the nodes thereby minimizing photon scattering, and which traps atoms deep in the Lamb-Dicke regime (see Fig. 1). The "transverse" beams confine the atoms in tubes oriented along the $z$ direction and the "longitudinal" beams produce standing waves of $\sigma_{+}$and $\sigma_{-}$light within each tube. The polarization gradient of the longitudinal fields allows the distinction of two "types" of atoms: those trapped at the nodes of $\sigma_{+}$-polarized wells and those trapped at nodes of $\sigma_{-}$-polarized wells. Central to our method is the ability to vary the lattice geometry dynamically: changing the angle $\theta$ between the longitudinal lasers' polarizations varies the distance $\delta Z$ between the minima of these wells according to $k_{L} \delta Z=\tan ^{-1}(\tan \theta / 2)$. Two atoms trapped in neighboring wells can be brought into the same linearly polarized well by rotating the lasers' polarizations to parallel, adiabatically compared with the oscillation frequency in the well. For large detunings stimulated Raman transitions by the lattice lasers are suppressed and the atom maintains its internal state. Once in the same well, the atoms can be made to interact by applying an auxiliary "catalysis laser" that excites the atomic dipoles for a short time. A rotation of the laser polarization beyond $90^{\circ}$ slides the potential wells by more than a quarter wavelength. A given $\sigma_{+}$atom can thus interact sequentially with all other $\sigma_{-}$atoms, and an arbitrary entangled state within a tube can be created.

For each of the atomic types $\sigma_{ \pm}$, we define a computational basis, $|1\rangle_{ \pm},|0\rangle_{ \pm}$, of logical one and zero (see Fig. 2),

$$
\begin{aligned}
|1\rangle_{ \pm} & \equiv\left|F_{\uparrow}, M_{F}= \pm 1\right\rangle \otimes|n=0\rangle, \\
|0\rangle_{ \pm} & \equiv\left|F_{\downarrow}, M_{F}=\mp 1\right\rangle \otimes|n=0\rangle,
\end{aligned}
$$

where $F_{\uparrow, \downarrow}=I \pm 1 / 2$ denote the two hyperfine levels associated with the $S_{1 / 2}$ ground state and nuclear spin $I$ (half-integer), $M_{F}$ is the magnetic sublevel, and $|n=0\rangle$ is the vibrational ground state of the associated potential. Single-qubit operations can be performed via pulses that are Raman-resonant with one type of atom. Two-qubit operations involve conditioning the state of one atom on the state of the other. For example, a C-NOT can be performed in the following way. Two atoms are made to reside in the same well as described above, and a weak $\pi$-polarized catalysis-laser field, propagating in the $x$ - $y$ plane, is used to excite a near-resonant atomic dipole. If this laser is tuned to the $\left|S_{1 / 2}, F_{\uparrow}\right\rangle \rightarrow\left|P_{3 / 2}, F_{\max }^{\prime}\right\rangle$ resonance, where $F_{\max }^{\prime}=I+3 / 2$, a dipole is excited only if the atom is in the $\left|F_{\uparrow}\right\rangle$ state (i.e., the logical states $\left.|1\rangle_{ \pm}\right)$. The dipole-dipole interaction thus causes a shift only of the $|1\rangle_{-} \otimes|1\rangle_{+}$two-qubit state and has neither diagonal nor off-diagonal matrix elements between any of the other two-qubit basis states. If the $\sigma_{-}$type acts as the control bit and the $\sigma_{+}$acts as the target, a Raman $\pi$-pulse on the shifted $|1\rangle_{-} \otimes|1\rangle_{+} \leftrightarrow|1\rangle_{-} \otimes|0\rangle_{+}$ transition achieves a C-NOT with the usual truth table. The polarizations of the Raman lasers and an external magnetic field ensure that the pulse does not drive any other transition. Once the logic operation is executed the state of a register can be read out as in the ion trap [5], by applying first a sequence of Raman pulses to isolate the population of that register in the $\left|F_{\uparrow}\right\rangle$ hyperfine state, and then detecting the amount of fluorescence on the cycling transition $\left|S_{1 / 2}, F_{\uparrow}\right\rangle \rightarrow\left|P_{3 / 2}, F_{\text {max }}^{\prime}\right\rangle$.

The dipole-dipole interaction is dependent both on the internal electronic states of the atoms, which determine the tensor nature of the interaction, and on the motional states, which determine the atomic wave-function overlap with the dipole-dipole potential. In the low saturation limit, the excited states can be adiabatically eliminated and the dipole-dipole interaction Hamiltonian between a pair of atoms can be written as $H_{d d}=V_{d d}-i \hbar \Gamma_{d d}$, where $V_{d d}$ describes the level shift and $\Gamma_{d d}$ describes the enhancement of the spontaneous photon scattering rate due to cooperative effects. For dipoles induced by the $\pi$-polarized catalysis laser, $H_{d d}$ is diagonal in the computational subspace, with the only nonvanishing matrix element given by 14

$$
\left\langle 1_{+}, 1_{-}\left|H_{d d}\right| 1_{+}, 1_{-}\right\rangle=-\hbar \Gamma^{\prime} c_{g}^{4}\left\langle f\left(r, \theta_{r}\right)+i g\left(r, \theta_{r}\right)\right\rangle,
$$

where $c_{g}$ is the Clebsch-Gordan coefficient for the transition $\left|F_{\uparrow}, M_{F}= \pm 1\right\rangle \rightarrow\left|F_{\text {max }}^{\prime}, M_{F^{\prime}}= \pm 1\right\rangle$. The functions $f$ and $g$ describe the dependence of the dipole-dipole interaction on the relative position of the two atoms: $f+i g=i h_{0}^{(2)}(k r)+P_{2}\left(\cos \theta_{r}\right) i h_{2}^{(2)}(k r)$, where $h_{k}$ are spherical Hankel functions of order $k$, and $P_{2}(\mu)$ is the second-order Legendre polynomial. For small distances, $f$ scales as $1 / r^{3}$, whereas $g$ goes to unity, corresponding to the full cooperativity of the superradiant state. The figure of merit is then given by $\kappa \equiv\left\langle V_{d d}\right\rangle /\left\langle\hbar\left(c_{g}^{4} \Gamma^{\prime}+\Gamma_{d d}\right)\right\rangle=$ $-\left\langle f\left(r, \theta_{r}\right)\right\rangle /\left(1+\left\langle g\left(r, \theta_{r}\right)\right\rangle\right)$.

Though spherically symmetric wells maximize the radial wave-function overlap for atoms in their ground vibrational states, an isotropic relative coordinate wave function is orthogonal to the $Y_{2}^{0}$ dipole potential. We thus consider an axially symmetric harmonic potential with two atoms in the vibrational ground state, each described by an elliptical Gaussian wave packet with rms 
widths $x_{0} \neq z_{0}$. Figure 3 shows a plot of $\kappa$, calculated numerically, as a function $\eta_{\perp}=k x_{0}$ and $\eta_{\|}=k z_{0}$. Over the range of values shown, $\left\langle g_{00}\left(r, \theta_{r}\right)\right\rangle \approx 1$, i.e., full cooperativity. Given experimentally accessible localizations $x_{0}=\lambda / 60$ and $z_{0}=\lambda / 30$, corresponding to $\eta_{\perp} \approx 0.1$ and $\eta_{\|} \approx 0.2$, the figure of merit is $\kappa \approx-19.3$. This is sufficient to resolve the level shift and perform a twobit logic gate with reasonable fidelity. An approximate expression for $\kappa$, neglecting retardation effects, is

$\kappa \approx \frac{1}{8 \sqrt{\pi} \eta_{\perp}^{2} \eta_{\|}}\left[-2-3 \frac{\eta^{2}}{\eta_{\perp}^{2}}+3\left(\frac{\eta^{3}}{\eta_{\perp}^{3}}+\frac{\eta}{\eta_{\perp}}\right) \tan ^{-1}\left(\frac{\eta_{\perp}}{\eta}\right)\right]$

where $\eta^{-2} \equiv \eta_{\|}^{-2}-\eta_{\perp}^{-2}$. Keeping $\eta_{\perp}$ fixed and maximizing with respect to the ratio $\eta_{\|} / \eta_{\perp}$ gives $\kappa_{\max } \approx$ $-0.017 / \eta_{\perp}^{3}$ for a ratio $\left(\eta_{\|} / \eta_{\perp}\right)_{\max } \approx 2.18$. The small prefactor stems mainly from the fact the relative coordinate rms in $3 \mathrm{D}$ is at least $\sqrt{6}$ times the rms for a single particle in 1D.

An experiment to characterize the performance of the quantum gate can be performed as follows. Consider the measurement of the C-NOT truth-table on an ensemble of identical atomic pairs. After the atoms are cooled and the control and target atoms are prepared in one of the logical basis states, the gate operation is executed, and the four populations of the logical basis can be read out as described above. We can expect the occupation of atoms in an optical lattice to be random. Atoms without the appropriate neighbor are in principle unaffected by the two-bit gate operation but generally will contribute to measured populations and reduce the apparent fidelity of the gate. The background signal from unpaired atoms can be removed if we carry out additional measurements on a sample without any paired atoms, as well as measurements where the gate is operated once, population flushed from a register, and the gate operated a second time before readout.

As a concrete example, consider Cs atoms with lasers trapping blue of the ${ }^{6} S_{1 / 2} \rightarrow{ }^{6} P_{3 / 2}$ transition at $\lambda=$ $852 \mathrm{~nm}$. Given intensities of $100 \mathrm{~W} / \mathrm{cm}^{2}$ for all beams in Fig. 1 and detunings of $\Delta_{\perp} / 2 \pi \approx 120 \mathrm{GHz}$ and $\Delta_{\|} / 2 \pi \approx$ $2 \mathrm{THz}$ for the transverse and longitudinal lasers, respectively, we achieve the requisite localizations, $\eta_{\perp}=0.1$, $\eta_{\|}=0.2$ discussed above, corresponding to oscillation frequencies $\nu_{\mathrm{osc}, \perp} \approx 200 \mathrm{kHz}$ and $\nu_{\mathrm{osc}, \|} \approx 50 \mathrm{kHz}$. For these parameters, photon scattering from the lattice produces a homogeneous linewidth $\Gamma_{\text {lat }}^{\prime} / 2 \pi \approx 4 \mathrm{~Hz}$. The catalysis laser is chosen as a perturbation to the trapping potentials, e.g., $\left|V_{d d}\right| / h=5 \mathrm{kHz}$. Taking this field on atomic resonance and given a figure of merit $\kappa=\left|V_{d d}\right| / \Gamma_{\text {cat }}^{\prime}=19.3$, the required catalysis intensity is $I_{\text {cat }} \approx 0.1 \mu \mathrm{W} / \mathrm{cm}^{2}$, corresponding to a superradiant scattering linewidth $\Gamma_{\text {cat }}^{\prime} / 2 \pi \approx 250 \mathrm{~Hz}$. Though the level shift depends sensitively on the atomic localization, it is a relatively weak function of the laser intensity since $\eta \sim I^{1 / 4}$. These parameters should allow one to carry out a few logical operations with high fidelity.

Though optical lattices hold promise for producing entangled states of a few atoms, much remains to be done to implement even a rudimentary quantum computation. From the experimental side, one must develop a method for addressing and reading out the state of individual qubits, which are generally spaced closer than the optical wavelength. One possibility is to tag the atomic resonance to the position of a well by use of a gradient magnetic field or an additional ac-Stark shift as demonstrated in [16]. Alternatively, lattices could be designed with more widely separated wells through the superposition of many different wave vectors or the use of very long wavelength lasers (such as an intense $\mathrm{CO}_{2}$ laser 15]). A major theoretical issue is the effect of atomic collisions [17]. For small interatomic distances the intense bluedetuned lattice fields will be resonant with a molecular potential which may lead to inelastic processes. Even for atoms in the electronic ground state, long-range molecular potentials can play an important role in atom-atom interactions for densities corresponding to two atoms in the same well. Though such interactions might be destructive for inelastic hyperfine-changing collisions [18], elastic collisions might provide an alternative coherent coupling scheme with a lower decoherence rate [19], especially in the region of a Feschbach resonance that can be tuned with a magnetic field [20]. Another important question is that of error correction. For example, in our scheme each 1D "tube" of atoms constitutes a separate quantum register acting in parallel with all the others. One might capitalize on this massive parallelism to increase the error threshold for fault-tolerant computation. Optical lattices are extremely flexible, with many experimental "knobs", allowing a wide variety of possible mechanisms for implementing the essential features of quantum logic.

We thank Paul Alsing and John Grondalski for useful discussions. This work was supported in part by New Mexico Universities Collaborative Research (Grant No. 9769), the Office of Naval Research (Grant No. N0001493-1-0116), the National Science Foundation (Grant No. PHY-9503259), the Army Research Office (Grant No. DAAG559710165), and the Joint Services Optics Program (Grant No. DAAG559710116).

[1] A. M. Steane, Rep. Prog. Phys. 61, 117 (1998), and references therein.

[2] A. Steane, Proc. Roy. Soc. A 452, 2551 (1996); A. R. Caldebank and P. W. Shor, Phys. Rev. A 54, 1098 (1996).

[3] J. Preskill, Proc. Roy. Soc. A, 454385 (1998); E. Knill, R. Laflamme, and W. Zurek, Science 279, 342 (1998). 
[4] J. I. Cirac and P. Zoller, Phys. Rev. Lett. 74, 4094 (1995).

[5] C. Monroe et al., Phys. Rev. Lett. 75, 4714 (1995).

[6] D. Wineland et al., Forschr. Phys. 46, 363 (1998).

[7] B. E. King et al., Phys. Rev. Lett. 81, 1525 (1998). J. F. Poyatos, J. I. Cirac and P. Zoller, ibid. 1322. D. F. V. James, ibid. 317.

[8] N. A. Gershenfeld and I. L. Chuang, Science 275, 350 (1997).

[9] P. Domokos et al., Phys. Rev. A 52, 3554 (1995); Q. A. Turchette et al., Phys. Rev. Lett. 75, 4710 (1995).

[10] D. Loss and D. P. DiVincenzo, Phys. Rev. A 57, 120 (1998).

[11] B. E. Kane, Nature 393, 133 (1998).

[12] P. S. Jessen and I. H. Deutsch, Adv. Atom. Mol. Opt. Phys. 36, 91 (1996), and references therein.

[13] S. E. Hamann et al., Phys. Rev. Lett 80, 4149 (1998); H. Perrin et al., Europhys. Lett. 42, 395 (1998).

[14] E. V. Goldstein, P. Pax, and P. Meystre, Phys. Rev. A 53, 2604 (1996); J. Gou and J. Cooper, Phys. Rev. A 51, 3128 (1995).

[15] S. Friebel et al., Phys Rev A 57, R20 (1998).

[16] J. R. Gardner et al., Phys. Rev. Lett. 70, 3404 (1993).

[17] P. D. Lett, P. S. Jullienne, and W. D. Phillips, Ann. Rev. Phys. Chem. 46, 423 (1995).

[18] E. Tiesinga et al., Phys. Rev. A. 43, R5188 (1991).

[19] D. Jaksch et al., unpublished; LANL e-print archive: quant-ph/9810087

[20] E. Tiesinga, B. J. Verhaar, and H. T. C. Stoof Phys. Rev. A. 47, 4114 (1993).

FIG. 1. Schematic of a 3D blue detuned optical lattice. Two pairs of $\pi$-polarized beams $k_{\perp}$ provide transverse confinement, and the beams $k_{\|}$(at a different frequency) provide longitudinal confinement in $\sigma_{+}$and $\sigma_{-}$standing waves. The solid (dotted) contours represent the resulting ellipsoidal potential wells associated with $\sigma_{+}\left(\sigma_{-}\right)$polarization, separated pairwise by $\delta Z$, as a function of the relative polarization angle $\theta$.

FIG. 2. Schematic energy levels for the D2 line of a generic alkali in the presence of a small longitudinal magnetic field (not to scale). The computational basis states for atoms that follow $\sigma_{ \pm}$light are indicated. The catalysis laser $\omega_{c}$ is near resonant for $|1\rangle_{ \pm}$states. The transverse and longitudinal trapping frequencies $\omega_{\perp}$ and $\omega_{\|}$are detuned very far to the blue of resonance. Unitary manipulation via Raman pulses connecting only the $|0\rangle_{+}$and $|1\rangle_{+}$states is shown.

FIG. 3. Plot of $\kappa$, the ratio of the coherent dipole-dipole level shift to the total linewidth, as a function of the Lamb-Dicke localization parameter in the transverse direction, $\eta_{\perp}$, and the longitudinal direction, $\eta_{\|}$. 


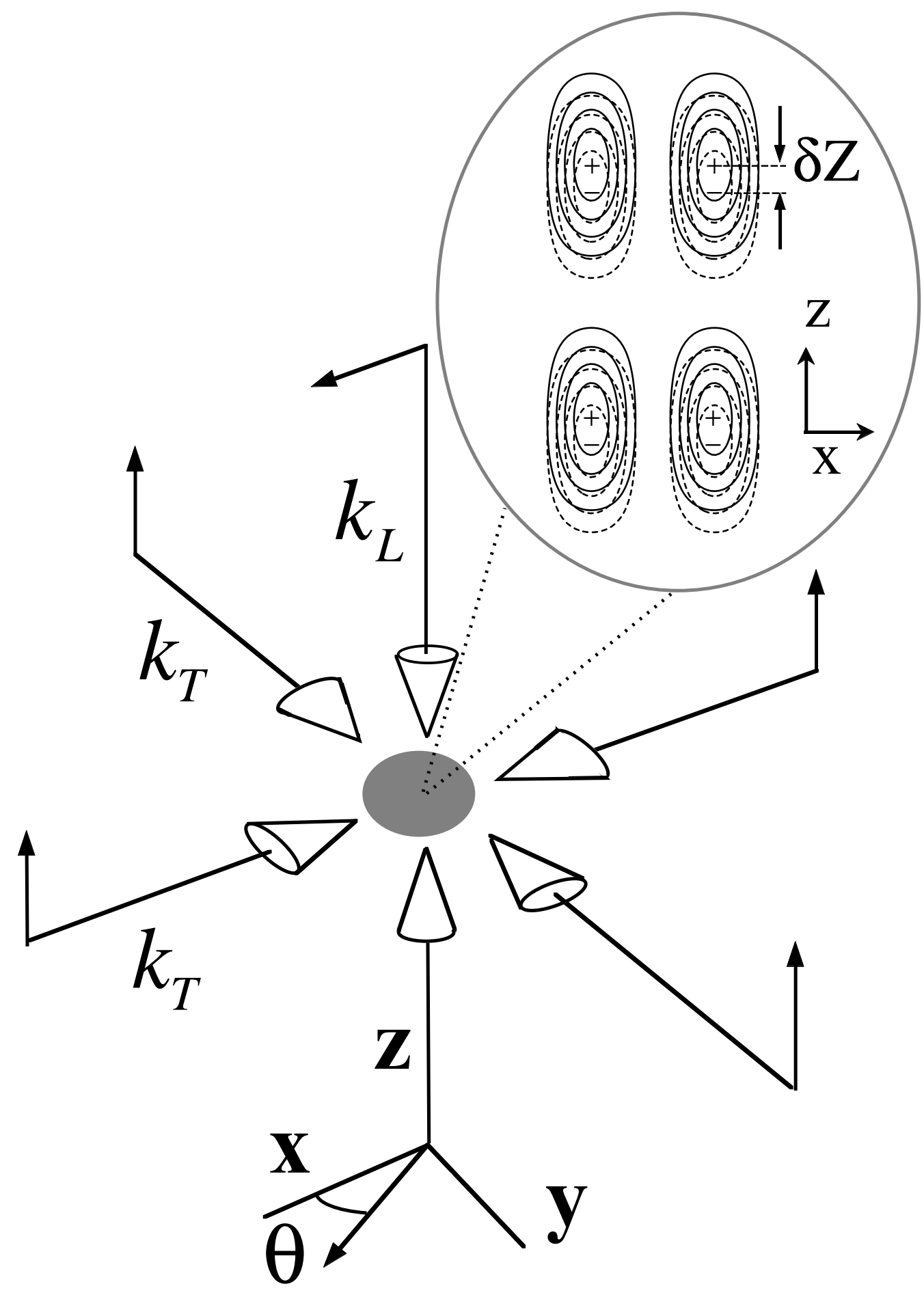




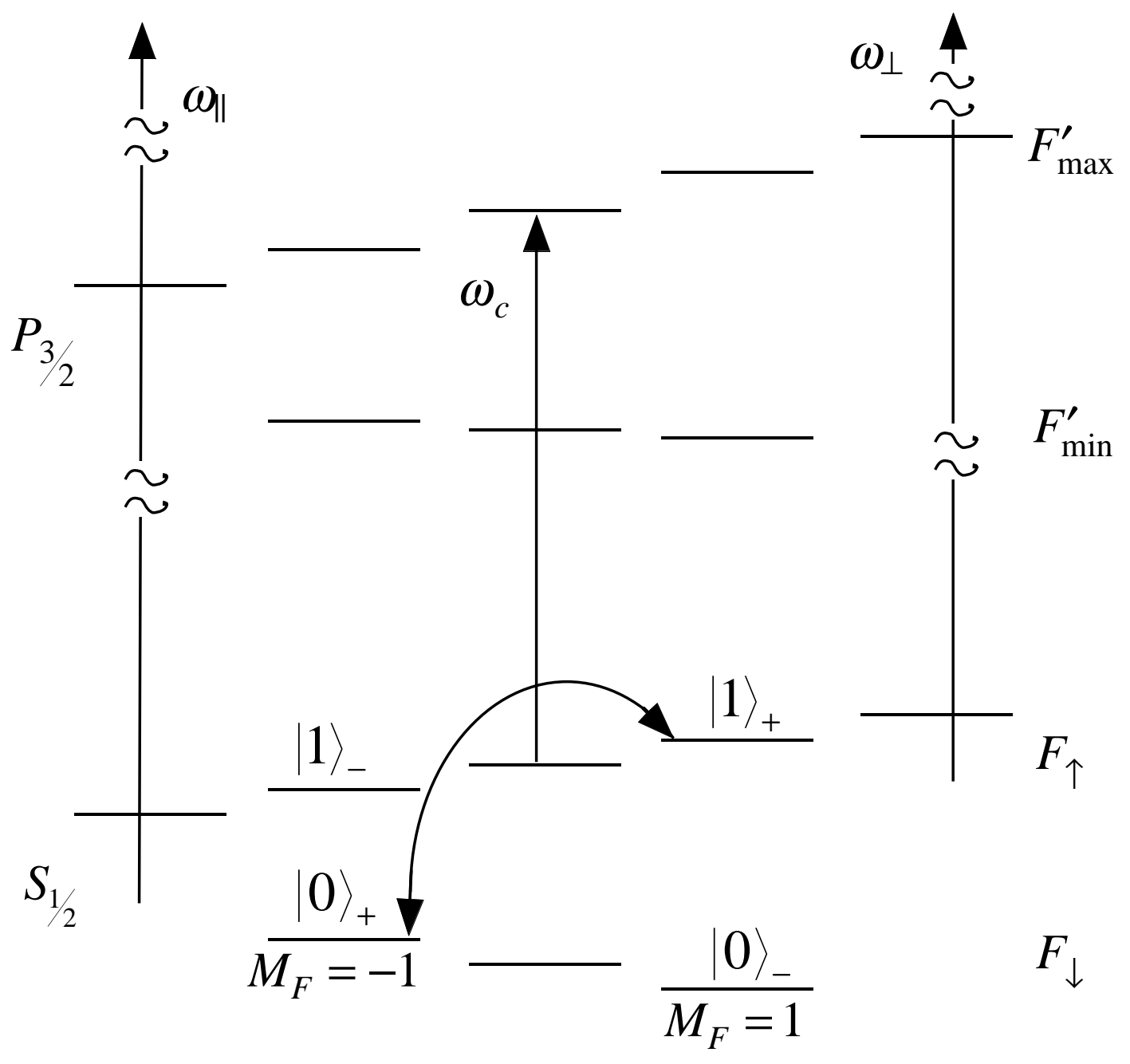




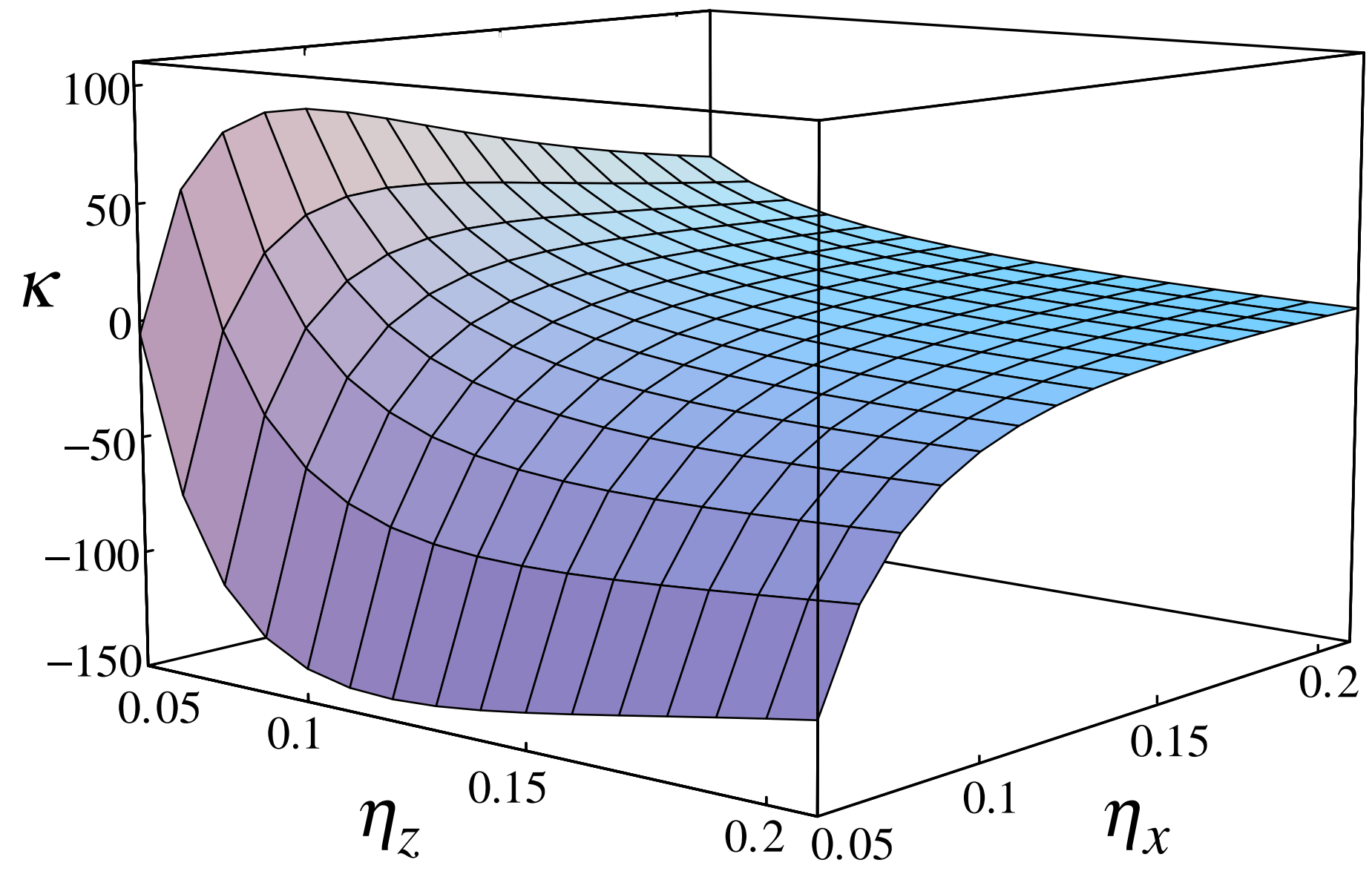

